\title{
Nouvelles espèces du genre Ptilonyssus (Rbinonyssidae, Parasitiformes) parasites de la cavité nasale de la Sittelle Sitta europaea coesia Wolf
}

\author{
par Z. FEIDER et Iulia MIRONESCU \\ [Laboratoire de Zoologie, Université «Al. I. Cuza», Iasi, R.S. de Roumanie]
}

\section{Résuméé}

Les auteurs décrivent quatre nouvelles espèces de la famille Rhinonyssidae: Ptilonyssus strandtmannianus, Ptilonyssus tribaspis, Ptilonyssus bregetovae et Ptilonyssus pelmaspis, qui parasitent les cavités nasales de la sittelle.

\section{Summary}

The authors described four new species from the family Rhinonyssidae: Ptilonyssus strandtmannianus, Ptilonyssus tribaspis, Ptilonyssus bregetovae and Ptilonyssus pelmaspis, parasitizing the nasal cavities of the bird Sitta europaea coesia Wolf.

Les Rhinonyssides parasites dans la cavité nasale de la sittelle sont peu connus. Pour la première fois, en 1963 et 1965 , Fain a décrit Ptilonyssus sittae, la seule espèce connue parasite sur la sittelle (5).

En ce qui concerne le genre Ptilonyssus Berlese et Trouessart, 1889, il est caractérisé par les boucliers dorsaux, en nombre de deux ou trois d'après Fain. D'après Vitzthun, Strandtmann et Bregetova (1) seulement les espèces pourvues de deux boucliers appartiennent au genre Ptilonyssus, tandis que les espèces avec trois boucliers dorsaux sont classées dans un nouveau genre Ptilonyssoides Vitzthum, 1935. 
En considérant l'observation de Fain (2) comme juste, selon laquelle le nombre des boucliers dorsaux n'a pas une valeur générique, nous considérons que les espèces avec trois boucliers dorsaux appartiennent aussi au genre Ptilonyssus.

Les quatre nouvelles espèces que nous avons trouvé sont: Ptilonyssus strandtmannianus n. sp., dédiée à l'acarinologue R. W. Strandtmann, P. tribaspis n. sp. ( $\alpha \sigma \pi i \zeta=$

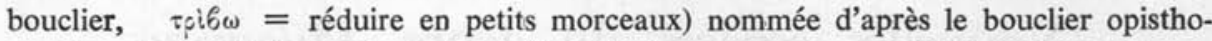
somal qui est divisé en plusieurs parties, $P$. bregetovae $\mathrm{n}$. sp., dédié à l'acarinologue N. Bregetova et $P$. pelmaspis n. sp., $(\pi \xi \lambda \mu \alpha=$ semelle, $\alpha \sigma \pi \iota \zeta=$ bouclier $)$ nommée d'après la forme du bouclier opisthosomal. Elles s'ajoutent au nombre des autres neuf espèces de Ptilonyssus pourvues de trois boucliers, parasites sur divers oiseaux (3), (4), (5), (6), (7).

Le nombre des spécimens*, les localités et la date de collections sont notés dans le tableau I.

\section{Tableau I}

COLLECTIONS DE Rhinonyssidae PARASITES SUR Sitta europaea

\begin{tabular}{|c|c|c|c|c|c|c|c|}
\hline $\begin{array}{l}\text { : } \\
\stackrel{0}{0} \\
\stackrel{0}{0} \\
0\end{array}$ & 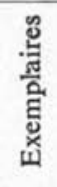 & $q$ & $\sigma^{*}$ & 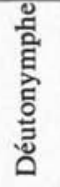 & Localité & Date & $\begin{array}{c}\text { Nouvelle espèce de } \\
\text { Ptilonyssus }\end{array}$ \\
\hline $1 \ldots$ & 8 & 6 & 1 & 1 & Gura-Humorului & 26-XI-1950 & strandtmannianus \\
\hline $2 \ldots$ & 2 & 1 & 1 & 一 & Repedea, Iasi & 26- I-1961 & strandtmannianus \\
\hline $3 \ldots$ & 2 & 2 & 一 & - & Gura Humorului & 6-III-1966 & tribaspis \\
\hline $4 \ldots$ & 2 & 2 & - & 一 & Zamora, Sinaia & 24-II-1959 & $\begin{array}{l}\text { pelmaspis } \\
\text { Sregetovae }\end{array}$ \\
\hline $5 \ldots$ & 1 & 1 & - & - & Sinaia & 8-II-1963 & Sregetovae \\
\hline Total . & 15 & 12 & 2 & 1 & & & \\
\hline
\end{tabular}

Du tableau résulte que chaque espèce parasite a été trouvée dans un hôte distinct, à l'exception de la collection de Sinaia-Zamora, où dans le même hôte on a trouvé $P$. pelmaspis et $P$. bregetovae. Dans deux localités (Sinaia et Gura Homorului) nous avons trouvé deux espèces.

\section{Dimensions.}

Celles-ci penmettent de séparer les quatre espèces et aussi de différencier les deux sexes (tableau 2).

* Quelques oiseaux nous ont été procurés par $\mathbf{M}^{\mathrm{me}} \mathbf{R}$. Klüger de Jassy et St. Negru de Bucarest, que nous remercions à cette occasion. 


\section{Tableau II}

Dimensions du corps, en microns, des Quatre espèces De Ptilonyssus de LA Roumanie ET DE L'EsPèce dE BelgiQue

\begin{tabular}{|c|c|c|c|c|c|c|c|c|}
\hline \multirow{2}{*}{ Organes } & \multirow{2}{*}{ Espèce } & \multicolumn{3}{|c|}{ strandtmannianus } & tribaspis & 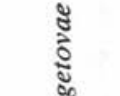 & 气ू̆ & sittae \\
\hline & & q & $\sigma^{*}$ & ปั & $q$ & q & $q$ & q \\
\hline \multicolumn{2}{|l|}{1} & 2 & 3 & 4 & 5 & 6 & 7 & 8 \\
\hline \multirow{2}{*}{ Idiosoma } & L & $537-660$ & $468-520$ & - & $573-758$ & $632-647$ & 592 & 605 \\
\hline & 1 & $259-268$ & $245-260$ & - & $280-333$ & $260-292$ & 296 & 325 \\
\hline \multirow{2}{*}{$\begin{array}{l}\text { Bouclier } \\
\text { podosomal }\end{array}$} & L & $199-227$ & $178-190$ & - & $216-260$ & $200-208$ & 224 & 210 \\
\hline & 1 & $157-182$ & $161-168$ & - & $196-216$ & $160-168$ & 184 & 162 \\
\hline \multirow{2}{*}{$\begin{array}{l}\text { Bouclier } \\
\text { opisthosomal }\end{array}$} & $\mathrm{L}$ & $87-100$ & $105-115$ & - & $38-40$ & 108 & 104 & 90 \\
\hline & 1 & $48-56$ & $70-77$ & - & $32-40$ & 48 & 52 & 48 \\
\hline \multirow{2}{*}{$\begin{array}{l}\text { Bouclier } \\
\text { pygidial }\end{array}$} & $\underline{L}$ & $64-80$ & $70-87$ & - & $68-$ & 72 & 76 & 72 \\
\hline & 1 & $59-72$ & $66-70$ & - & 68 & $64-68$ & 64 & 60 \\
\hline \multirow{2}{*}{$\begin{array}{l}\text { Bouclier } \\
\text { sternal }\end{array}$} & $\underline{L}$ & $100-120$ & 一 & - & $100-120$ & 94 & 108 & 90 \\
\hline & 1 & $77-92$ & - & - & $100-108$ & 88 & 81 & 90 \\
\hline \multirow{2}{*}{$\begin{array}{l}\text { Bouclier } \\
\text { sterno-génital }\end{array}$} & $\mathrm{L}$ & - & $210-217$ & - & - & - & - & - \\
\hline & $\overline{1}$ & - & $77-96$ & - & - & 一 & - & - \\
\hline \multirow{2}{*}{$\begin{array}{l}\text { Bouclier } \\
\text { génital }\end{array}$} & $\mathrm{L}$ & $119-144$ & - & - & 140 & 148 & 124 & 144 \\
\hline & 1 & $63-77$ & - & 一 & 72 & 68 & 61 & 60 \\
\hline \multirow{2}{*}{$\begin{array}{l}\text { Bouclier } \\
\text { anal }\end{array}$} & $\underline{L}$ & $80-88$ & $63-73$ & - & 84 & $73-88$ & 77 & 108 \\
\hline & 1 & $48-56$ & 52 & - & $48-56$ & $56-60$ & 54 & 54 \\
\hline \multirow{2}{*}{ Péritrème } & $\mathbf{L}$ & $32-38$ & 28 & 31 & 36 & 40 & 40 & 36 \\
\hline & 1 & $16-17$ & 14 & 10 & 16 & 16 & 14 & - \\
\hline \multirow{2}{*}{ Gnathosoma } & $\underline{\mathbf{L}}$ & $156-175$ & 129. & 129 & $168-188$ & 164-176 & 160 & 154 \\
\hline & 1 & $87-96$ & 30 & 59 & $92-100$ & $84-88$ & 69 & 90 \\
\hline \multirow{3}{*}{ Chélicère } & L & $136-154$ & 70 & 59 & 152 & $144-148$ & 124 & 150 \\
\hline & $\underline{L b}$ & $80-88$ & - & - & 88 & 88 & 65 & - \\
\hline & $\mathrm{lb}$ & $21-24$ & 21 & 17 & $22-24$ & 20 & 21 & 21 \\
\hline Doigt mobile & L & 8 & - & - & 6 & 8 & 8 & - \\
\hline \multirow{2}{*}{ Palpe } & $\underline{L}$ & $80-92$ & 70 & - & $84-88$ & 84 & 72 & - \\
\hline & 1 & $24-28$ & 25 & 14 & 28 & 24 & 24 & - \\
\hline
\end{tabular}

Abréviations : L : longueur ; 1 : largeur ; Lb: longueur du bulbe ; $\mathrm{lb}$ : largeur du bulbe. 


\begin{tabular}{|c|c|c|c|c|c|c|c|c|c|}
\hline \multirow{2}{*}{ Organes } & & \multirow{2}{*}{ Espèce } & \multicolumn{3}{|c|}{ strandtmannianus } & \multirow{2}{*}{$\begin{array}{c}\text { tribaspis } \\
\\
\Varangle\end{array}$} & \multirow{2}{*}{ 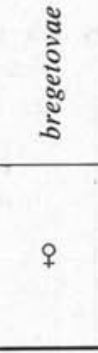 } & \multirow{2}{*}{ 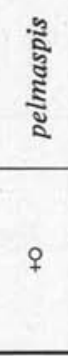 } & \multirow{2}{*}{$\begin{array}{r}\text { sittae } \\
\\
\text { t }\end{array}$} \\
\hline & & & q & $\sigma^{2}$ & 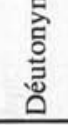 & & & & \\
\hline \multicolumn{3}{|c|}{1} & 2 & 3 & 4 & 5 & 6 & 7 & 8 \\
\hline \multirow{4}{*}{ Pattes } & & I & $314-372$ & 252- & - & $300-312$ & 300 & 300 & - \\
\hline & & II & $260-300$ & 310 & 210 & $244-248$ & 220 & 248 & - \\
\hline & & III & $252-280$ & $210-217$ & 210 & $244-252$ & 232 & - & - \\
\hline & & $\overline{\text { IV }}$ & $296-340$ & 252 & 227 & $284-300$ & 248 & 260 & - \\
\hline \multirow{8}{*}{ Coxes } & \multirow{2}{*}{ I } & $\mathrm{L}$ & $68-84$ & 63 & 63 & 72 & $76-88$ & 63 & - \\
\hline & & 1 & $60-70$ & 56 & 59 & 64 & $64-72$ & 53 & - \\
\hline & \multirow{2}{*}{ II } & $\underline{L}$ & $36-44$ & 35 & 35 & $40-44$ & $48-52$ & 44 & $\cdot-$ \\
\hline & & ] & $72-88$ & 70 & 70 & $84-96$ & $76-88$ & 67 & - \\
\hline & \multirow{2}{*}{ III } & L & $40-56$ & 35 & 28 & $44-52$ & $42-44$ & 44 & - \\
\hline & & 1 & $72-84$ & 73 & 70 & $88-92$ & $88-92$ & 80 & - \\
\hline & \multirow{2}{*}{ IV } & L & $40-52$ & 42 & 28 & $44-48$ & $48-52$ & 48 & - \\
\hline & & 1 & $77-84$ & 70 & 66 & 80 & $80-88$ & 72 & - \\
\hline \multirow{8}{*}{ Tarses } & \multirow{2}{*}{ I } & L & $70-80$ & 52 & - & 76 & $68-80$ & 76 & - \\
\hline & & 1 & $32-35$ & 28 & - & 32 & $32-36$ & 32 & - \\
\hline & \multirow{2}{*}{ II } & $\mathrm{L}$ & $56-76$ & 45 & 56 & $60-64$ & 72 & 60 & - \\
\hline & & 1 & $32-36$ & 28 & 35 & 32 & 36 & 36 & - \\
\hline & \multirow{2}{*}{ III } & L & $59-72$ & 45 & 59 & $60-64$ & - & - & - \\
\hline & & 1 & $32-36$ & 28 & 32 & 32 & - & - & - \\
\hline & \multirow{2}{*}{ IV } & L & $72-80$ & 59 & 63 & $72-80$ & - & 60 & - \\
\hline & & $\overline{1}$ & $32-42$ & 28 & 35 & 32 & - & 32 & - \\
\hline \multirow{4}{*}{ Griffes } & 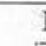 & I L & $38-48$ & 31 & - & $40-52$ & 40 & 36 & - \\
\hline & & II L & $40-49$ & 35 & 21 & $44-48$ & $40-52$ & 48 & - \\
\hline & & III $\mathrm{L}$ & $36-42$ & $35-38$ & 21 & $44-48$ & $40-44$ & 44 & - \\
\hline & & IV L & $38-49$ & 38 & 21 & 48 & $40-48$ & 44 & - \\
\hline
\end{tabular}

\section{Caractères morphologiques.}

Ptilonyssus strandtmannianus n. sp., est représenté par des femelles, des mâles et une déutonymphe, trouvés à Gura Humorului (Suceava) et à Repedea (Jassy).

Femelle: Le bouclier podosomal, avec le bord antérieur arrondi et les bords latéraux étranglés, de même que les autres boucliers dorsaux, est recouverts d'un 


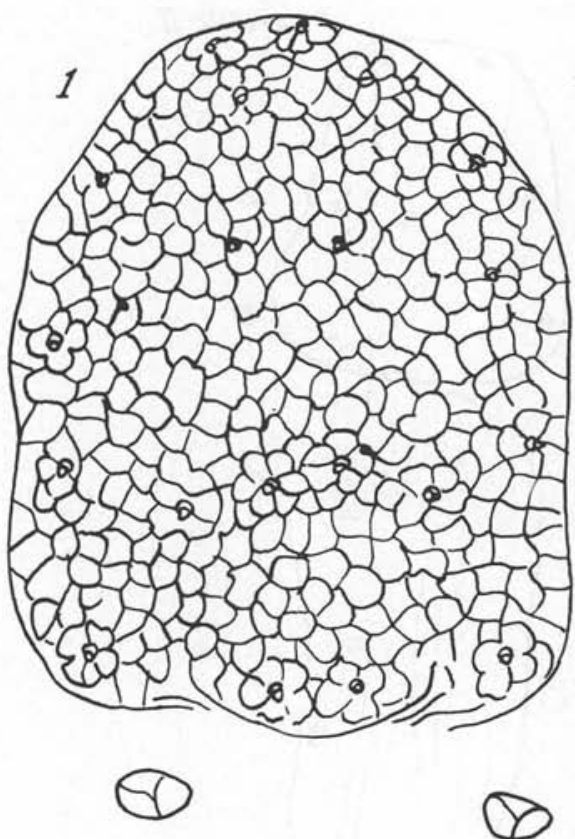

PLANCHE I
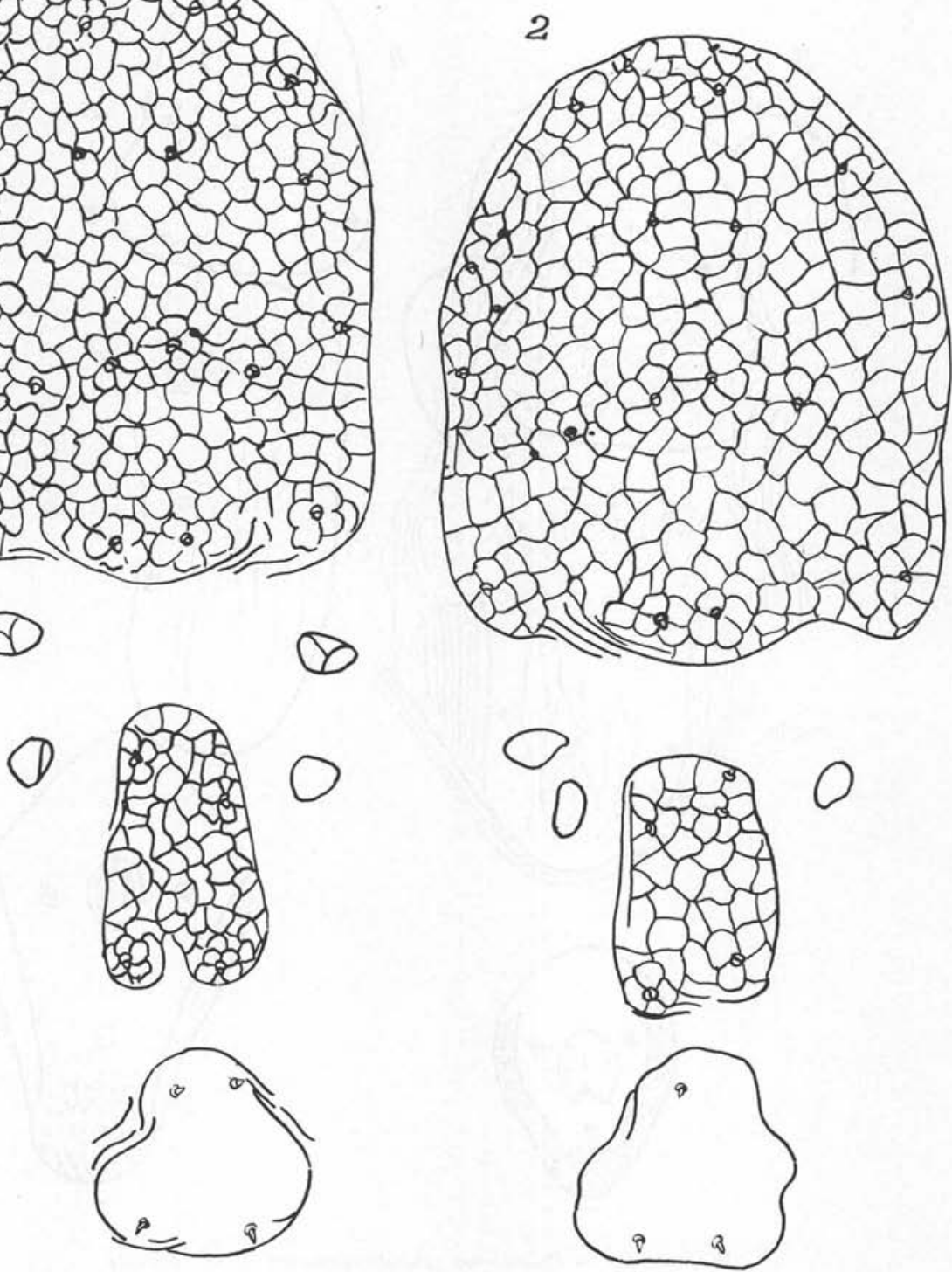

Planche I. - Ptilonyssus strandtmannianus n. sp., femelle

Fig. 1. - Boucliers dorsaux des spécimens de Gura humoru'ui

Fig. 2. - Boucliers dorsaux des spécimens de Repedea 

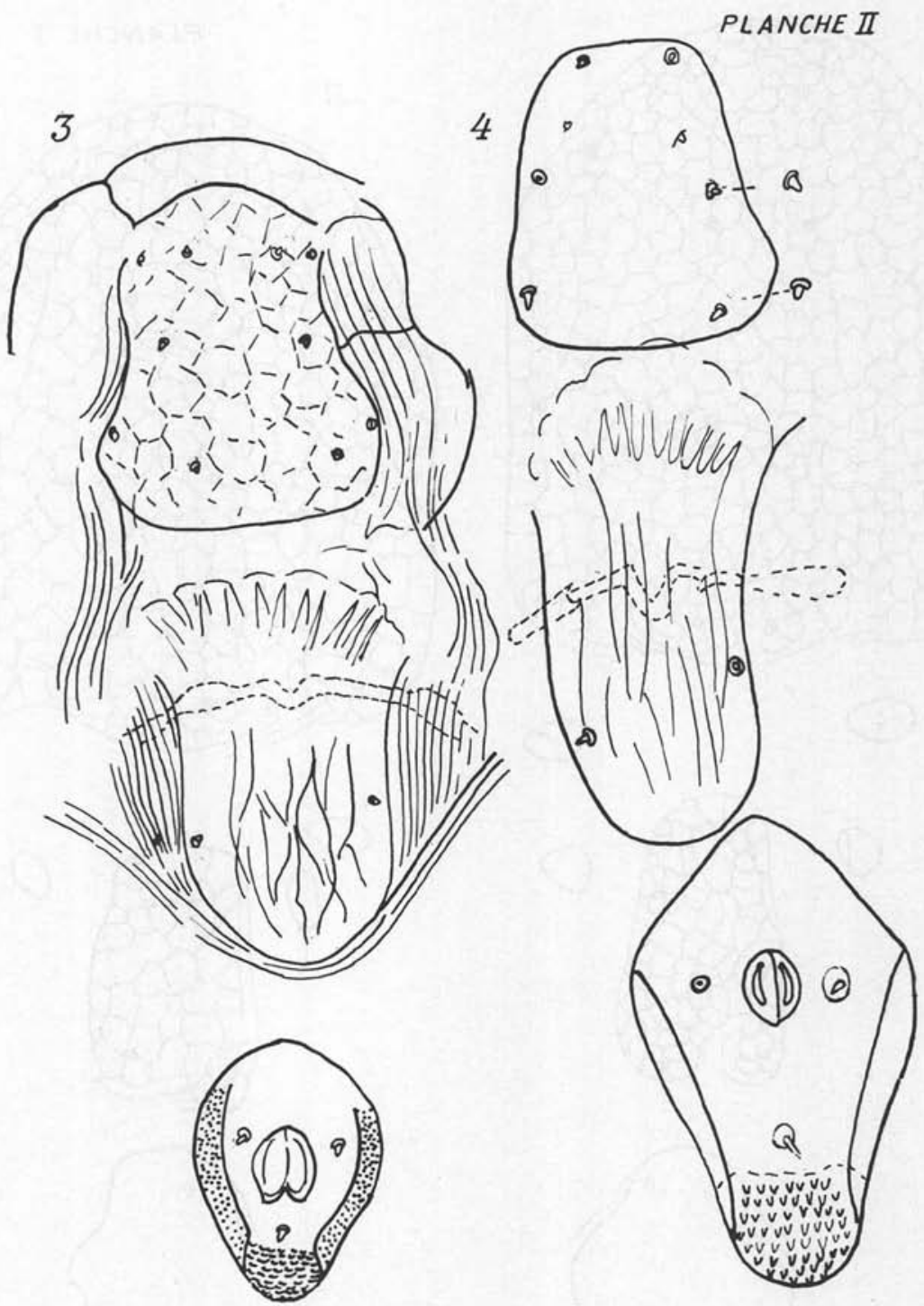

Planche II. - Ptilonyssus strandtmannianus n. sp., femelle

Fig. 3. - Boucliers ventraux des spécimens de Gura humorului

Fı́. 4. - Bouclier anal des spécimens de Repedea 


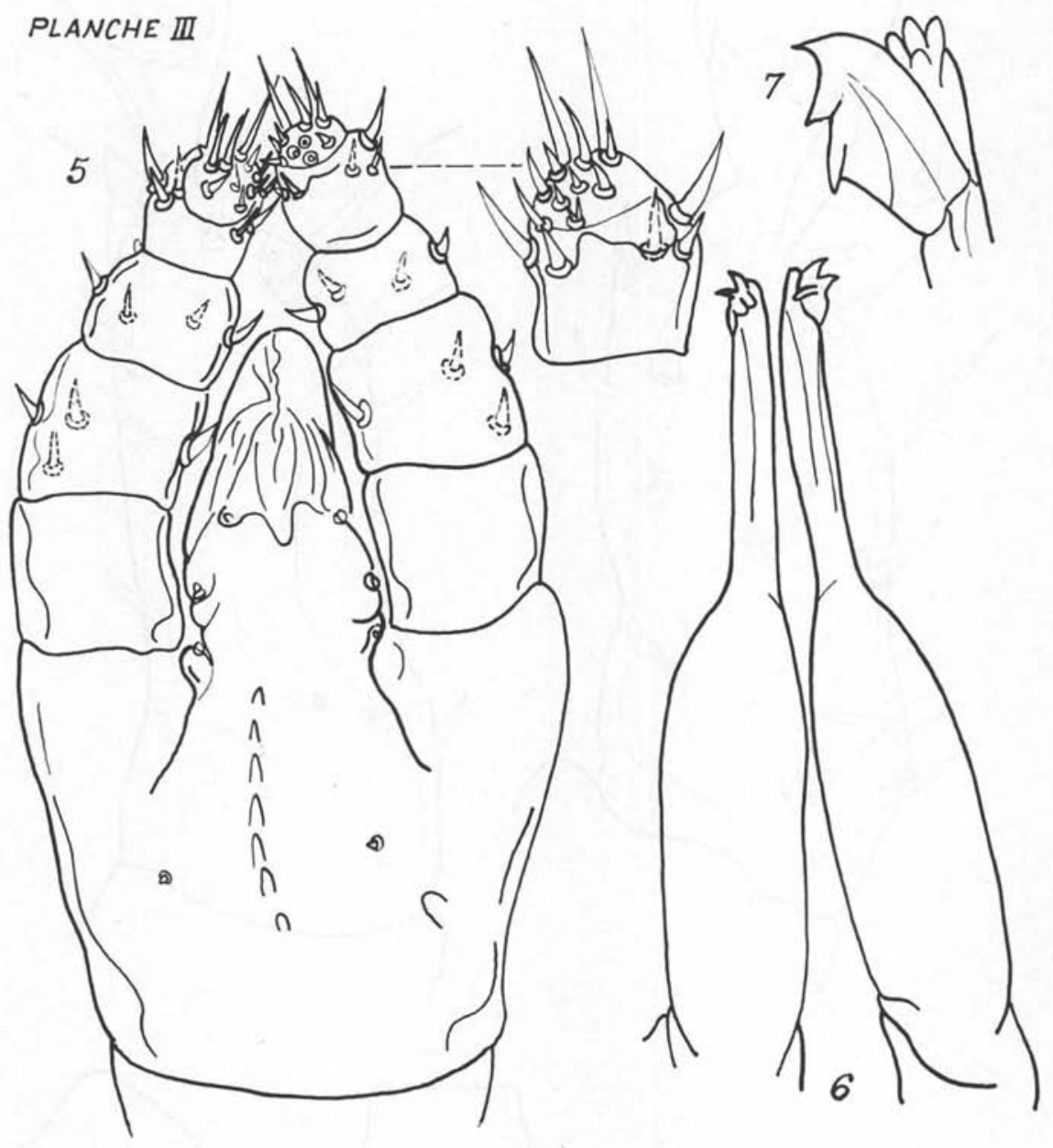

Planche III. - Ptilonyssus strandtmannianus n. sp., femelle

FiG. 5. - Gnathosoma, vue ventrale FIG. 6. - Chélicères

Fıg. 7. - Doigt mobile des chélicères. 
A
$\frac{1}{1}$
$\frac{1}{2}$
$\frac{1}{R}$
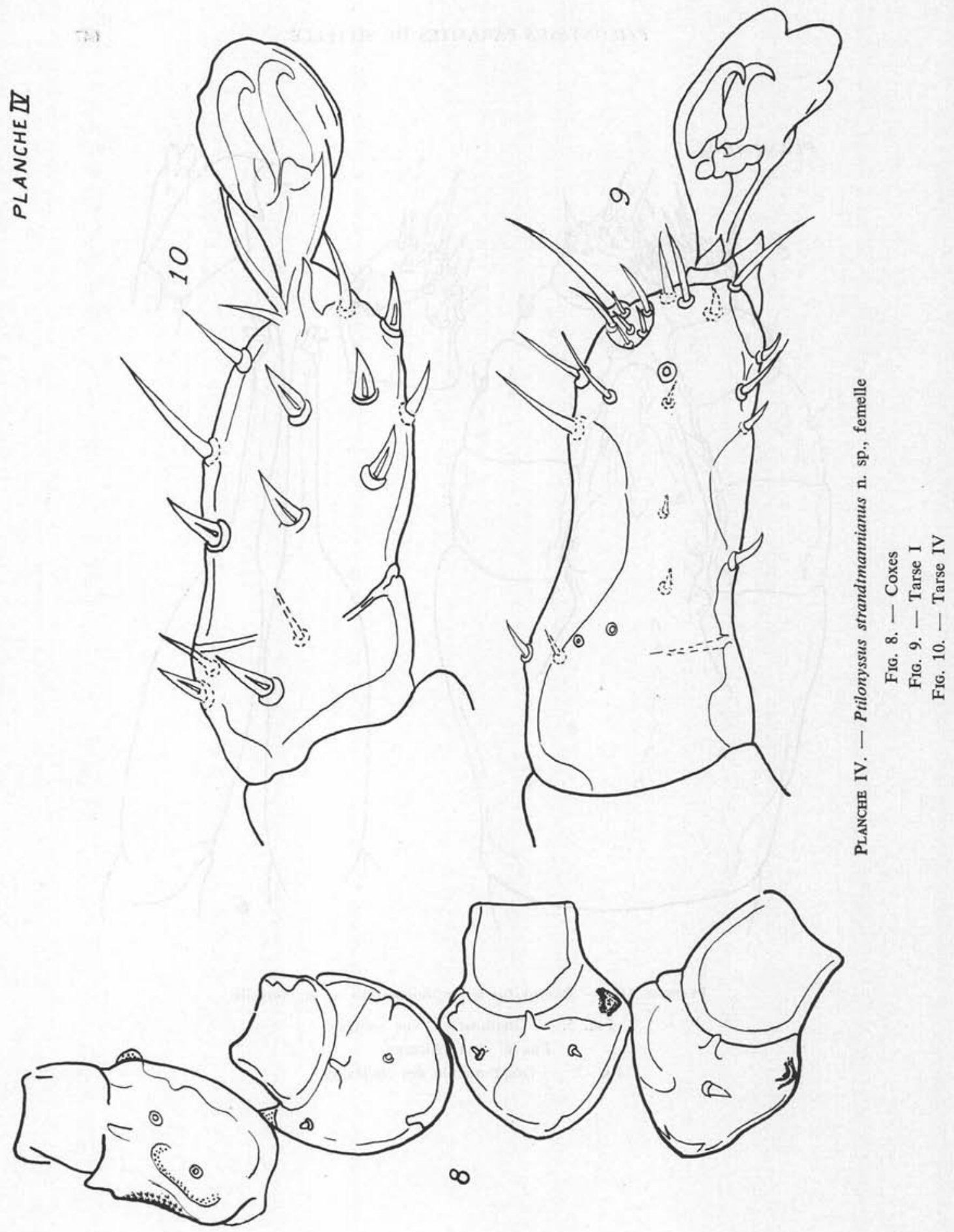

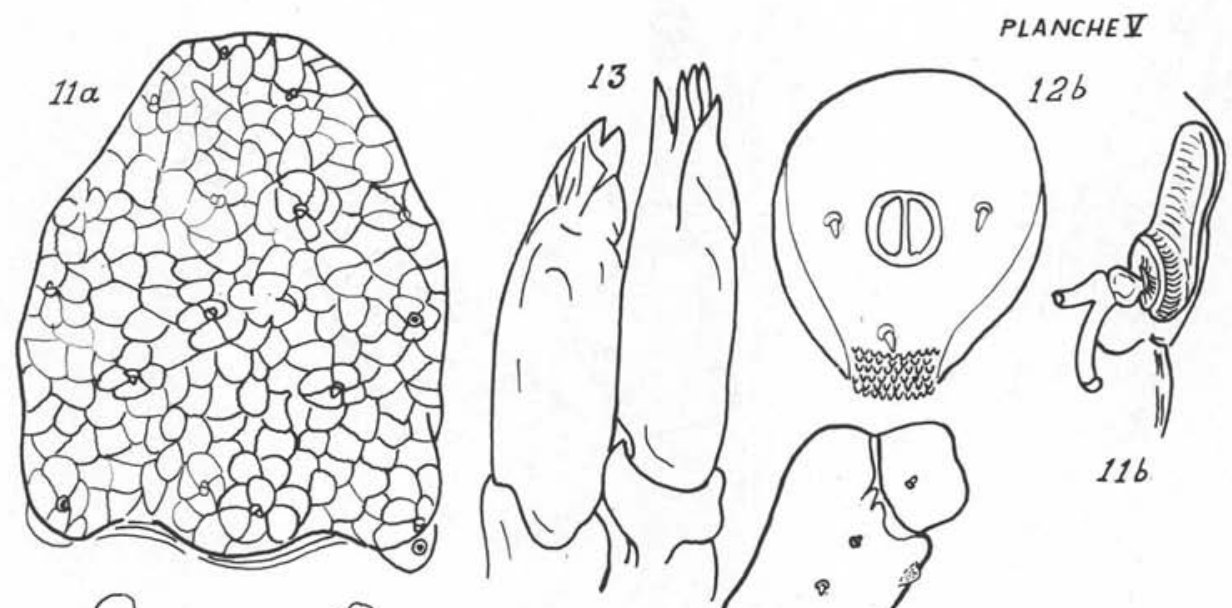

O
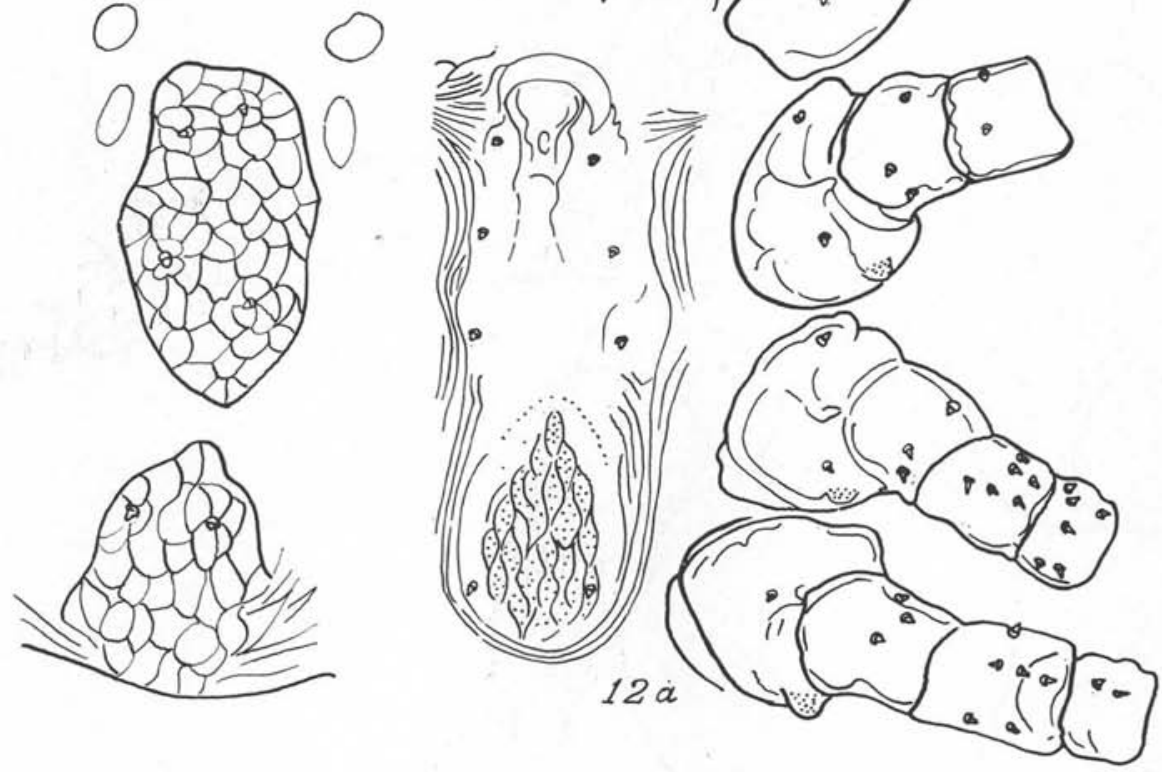

Planche V. - Ptilonyssus strandtmannianus n. sp., mâle

Fig. 11. $-a=$ vue dorsale, $b=$ péritrème

Fig. 12. $-a=$ bouclier sterno-génital et coxes, $b=$ bouclier anal

Fig. 13. - Chélicères 


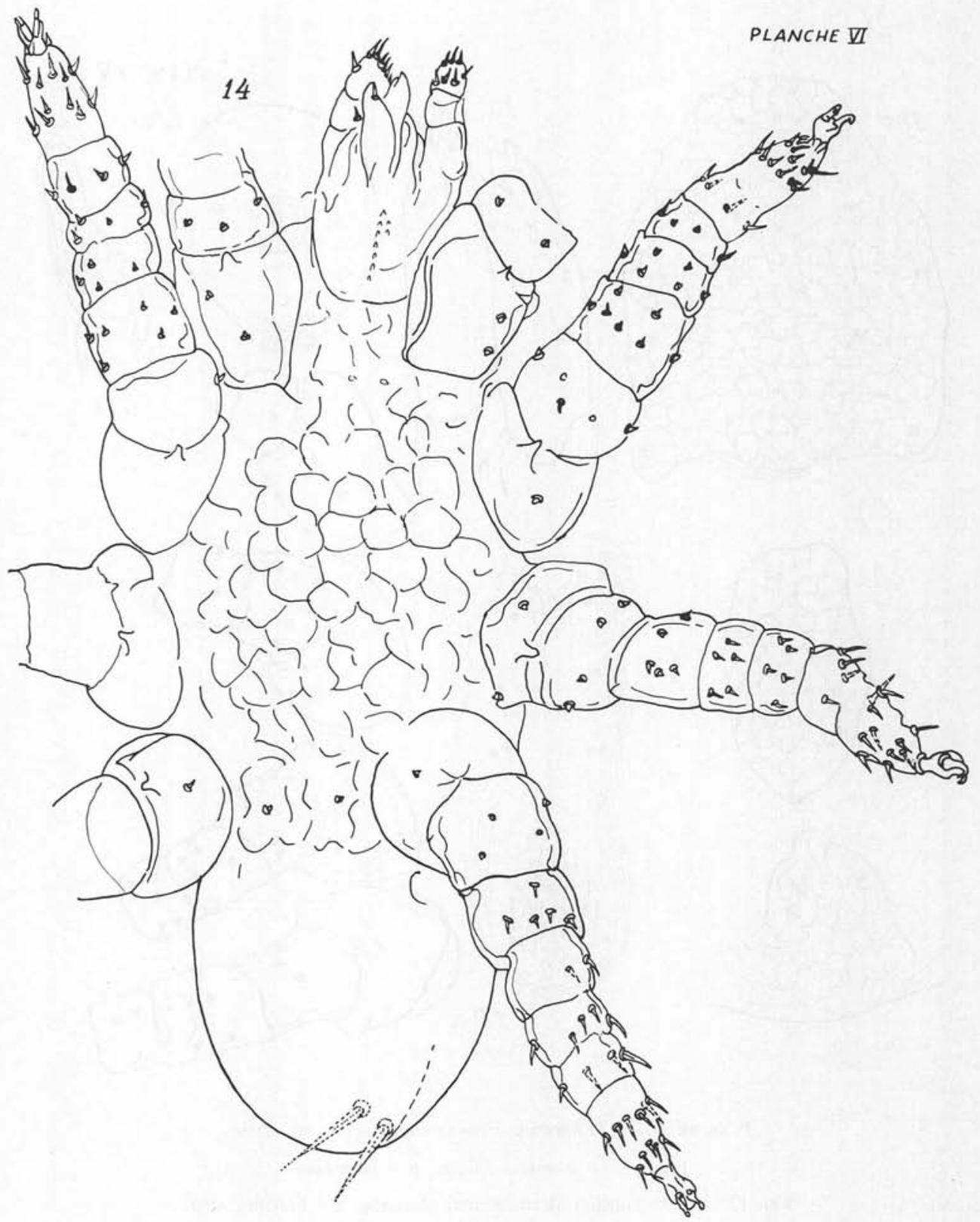

Planche VI. - Ptilonyssus strandtmannianus n. sp., déutonymphe Fig. 14. - Vue ventrale 
réseau à mailles équidimensionnelles, disposées en rosette autour des poils (fig. 1). Ce bouclier est plus large chez les spécimens de Repedea (fig. 2). Les 18 poils sont disposés en quatre rangées dont la troisième est en arrière de la moitié du bouclier. $\mathrm{A}$ l'extrémité postérieure le bouclier opisthosomal est divisé en deux prolongements asymétriques. Ces derniers sont moins visibles chez les spécimens de Repedea. Sur les côtés de ce bouclier se trouvent deux petits boucliers accessoires dépourvus de poils. Le bouclier pygidial de forme triangulaire est plus large chez les spécimens de Repedea.

Le bouclier sternal a une forme hexagonale chez les spécimens de Gura Humorului (fig. 3) et une forme plutôt trapezoïdale chez les spécimens de Repedea. Le bouclier génital, est élargi antérieurement, où il présente des rayons divergents. Le bouclier anal, avec un large cribum, a les valves anales en position centrale, et la paire de poils fixée au niveau de l'extrémité antérieure des valves. Les spécimens de Repedea ont les valves anales déplacées antérieurement (fig. 4).

Le gnathosoma a le deutérosternum formé de sept denticules (fig. 5). Les chélicères, avec le bulbe plus long, ont le doigt mobile pourvu de deux denticules latérales et un denticule étroit, dirigé en arrière (fig. 6 et 7). Parmi les neufs poils du palpotarse, huit sont spiniformes et un est bifide (fig. 5).

La première coxa est trapézoïdale, plus étroite vers l'extrémité médiale, tandis ques les autres coxae sont demi-lunaires (fig. 8). Le tarse I a six poils simples sur le bouton sensitif (fig. 9) tandis que le tarse IV a des poils aplatis sur la face inférieure (fig. 10).

Mâle : A la partie dorsale, le bouclier opisthosomal, à la différence de la femelle, est dépourvu de l'incision postérieure (fig. 11). Le bouclier sterno-génital, avec l'extrémité postérieure arrondie, a un large orifice génital. Le bouclier anal est plus arrondi que chez la femelle (fig. 2).

Les chélicères présentent plusieurs lobes antérieurs (fig. 13). Les coxae ont la même forme que chez la femelle.

DÉutonYMPHE. Les boucliers sont peu visibles, tandis que les poils pygidiaux, très longs et pourvus de petits barbules, se voient facilement. Les chélicères et les coxae sont comme chez l'adulte (fig. 14).

Holotype et paratypye, femelles, holotype mâle et deutonymphe chez les auteurs. Terra typica: Gura Humorului, (Suceava). Date : 26-XI-1950.

Ptilonyssus tribaspis n. sp. Femelle. Le bouclier podomomal, excavé antérieurement, est recouvert d'un réseau à mailles équidimensionnelles peu évidentes. Les 16 poils sont disposés en cinq rangées dont la quatrième est située en arrière de la moitié. Le bouclier opisthosomal est fragmenté en quatre segments arrondis pourvus chacun d'un seul poil (fig. 15). Le bouclier pygidial, trapézoïdal, présente les bords latéraux plus excavés que l'espèce précédente.

Le bouclier sternal, avec les bords latéraux presque parallèles, est pourvu de poils plus forts que l'espèce précédente. Le bouclier génital, étroit, est recouvert d'un réseau à mailles longitudinales et de points serrés. Le bouclier anal, avec la paire de 


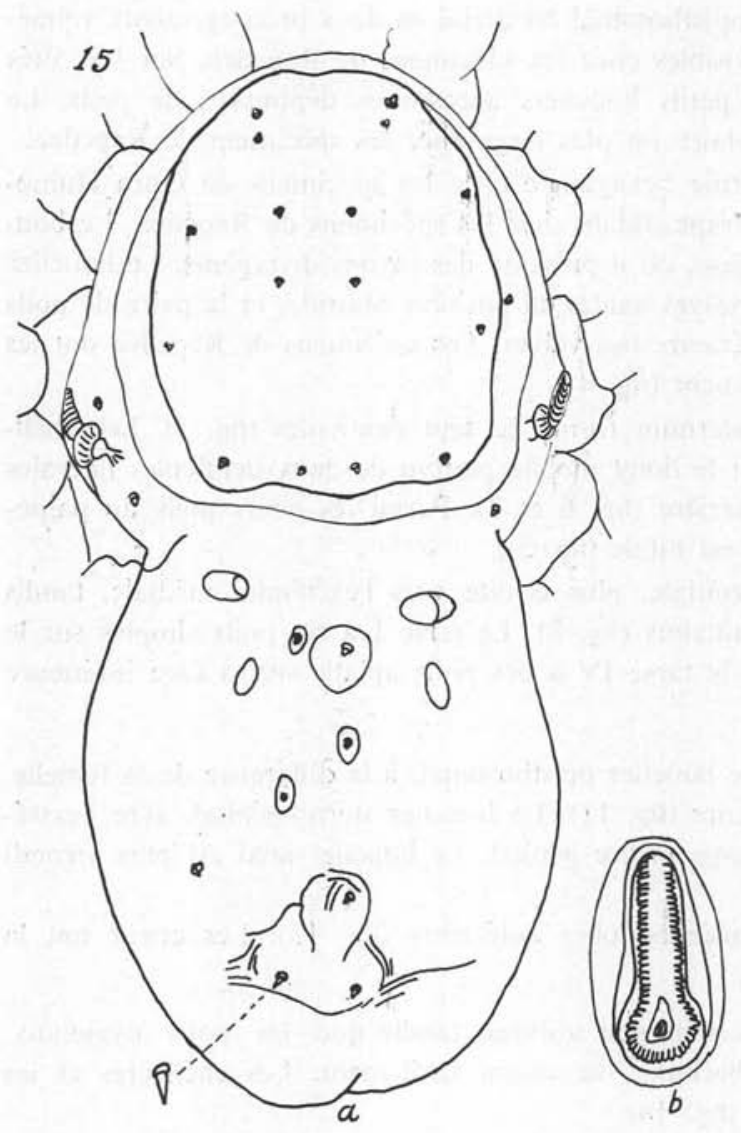

PLANCHE III

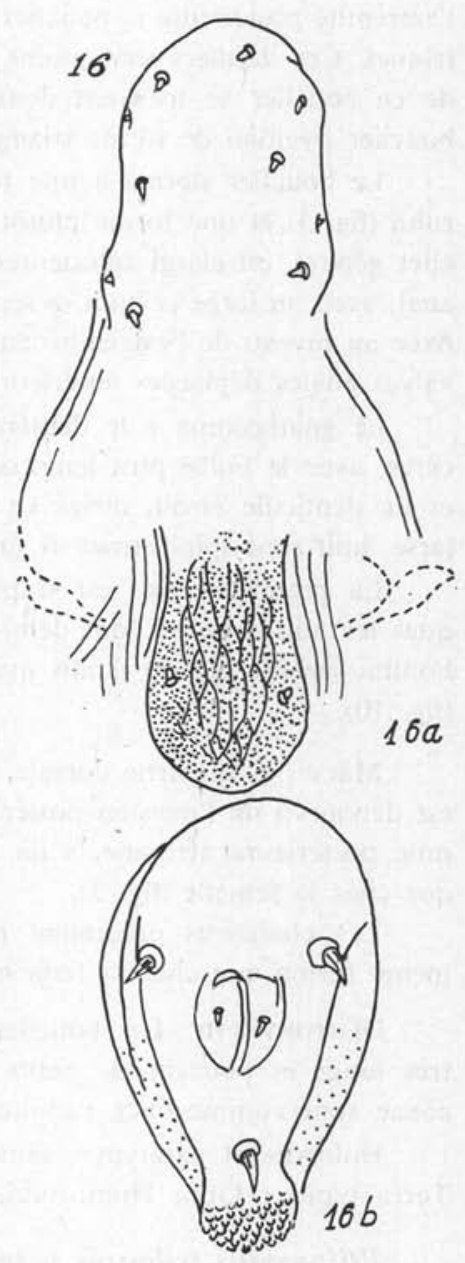

Planche VII. - Ptilonyssus tribaspis n. sp., femelle

Fig. 15. $-a=$ vue dorsale, $b=$ péritrème

FIg. 16. $-a=$ bouclier sternal, génital, $b=$ bouclier anal 

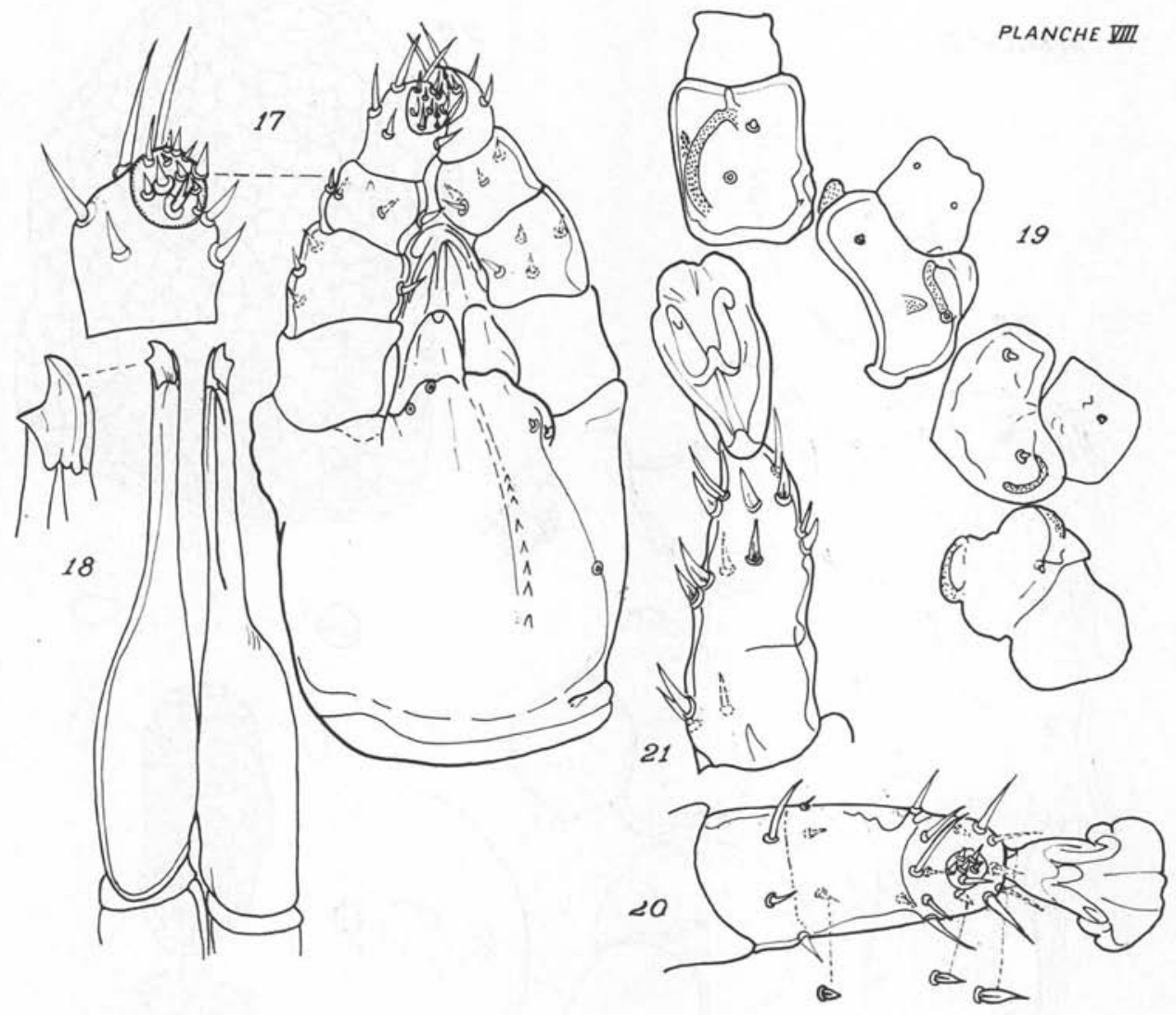

Planche VIII.- Ptilonyssus tribaspis n. sp., femelle

Fig. 17. - Gnathosoma, vue dorsale

FIG. 18. - Chélicères

Fig. 19. - Coxes

Fig. 20. - Tarse I

FIG. 21. - Tarse IV 
PLANCHE $\mathbb{X}$
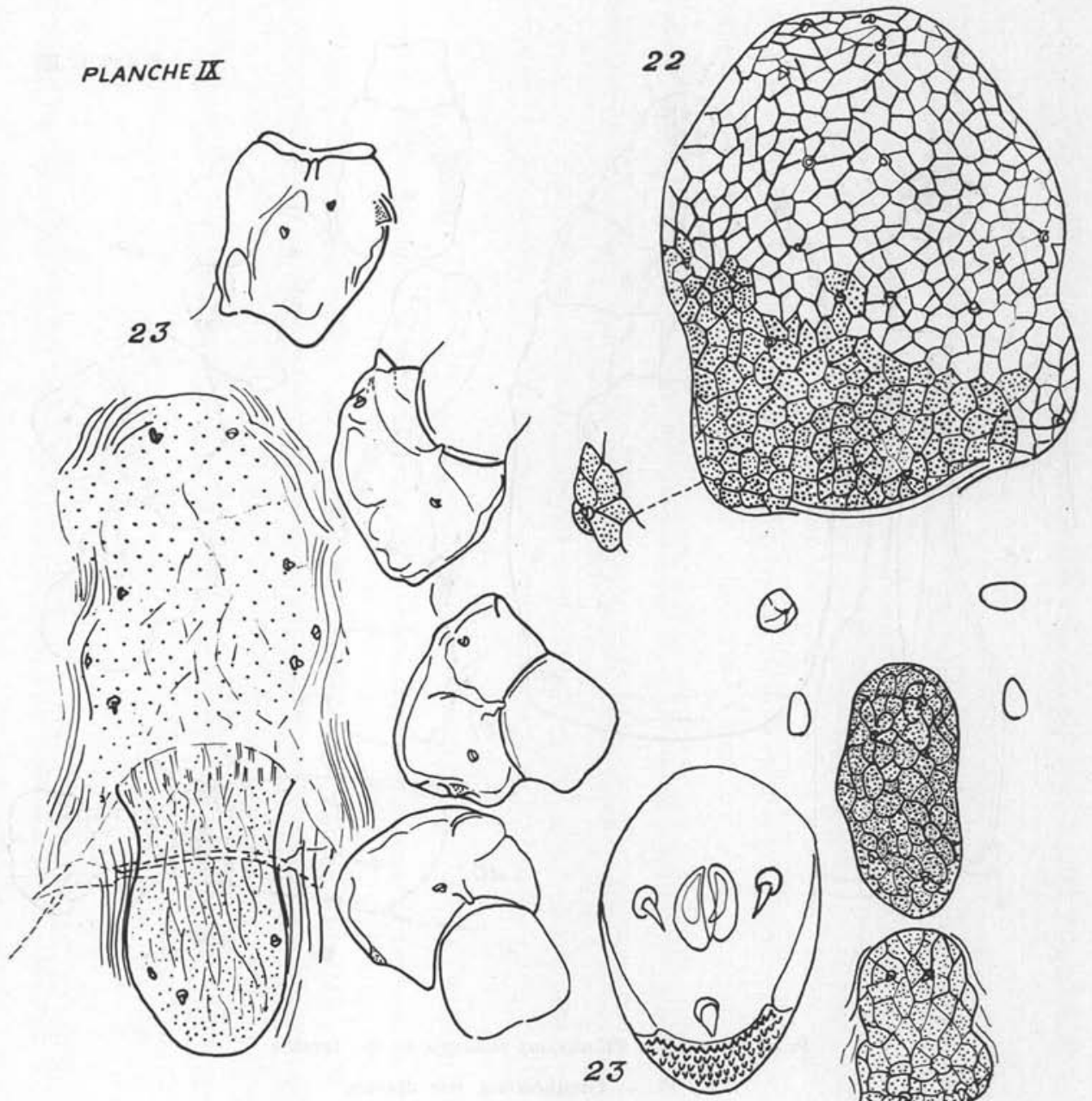

$\Theta$
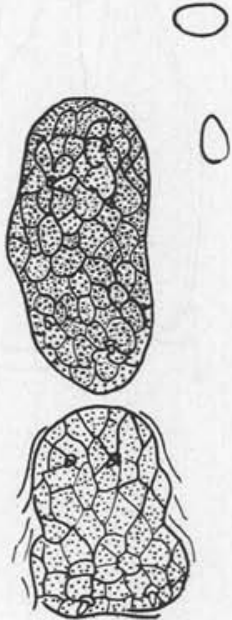

0

Planche IX. - Ptilonyssus bregetovae n. sp., femelle

Fig. 22. - Boucliers dorsaux

Fig. 23. - Boucliers ventraux et coxes 
PLANCHE $\bar{X}$

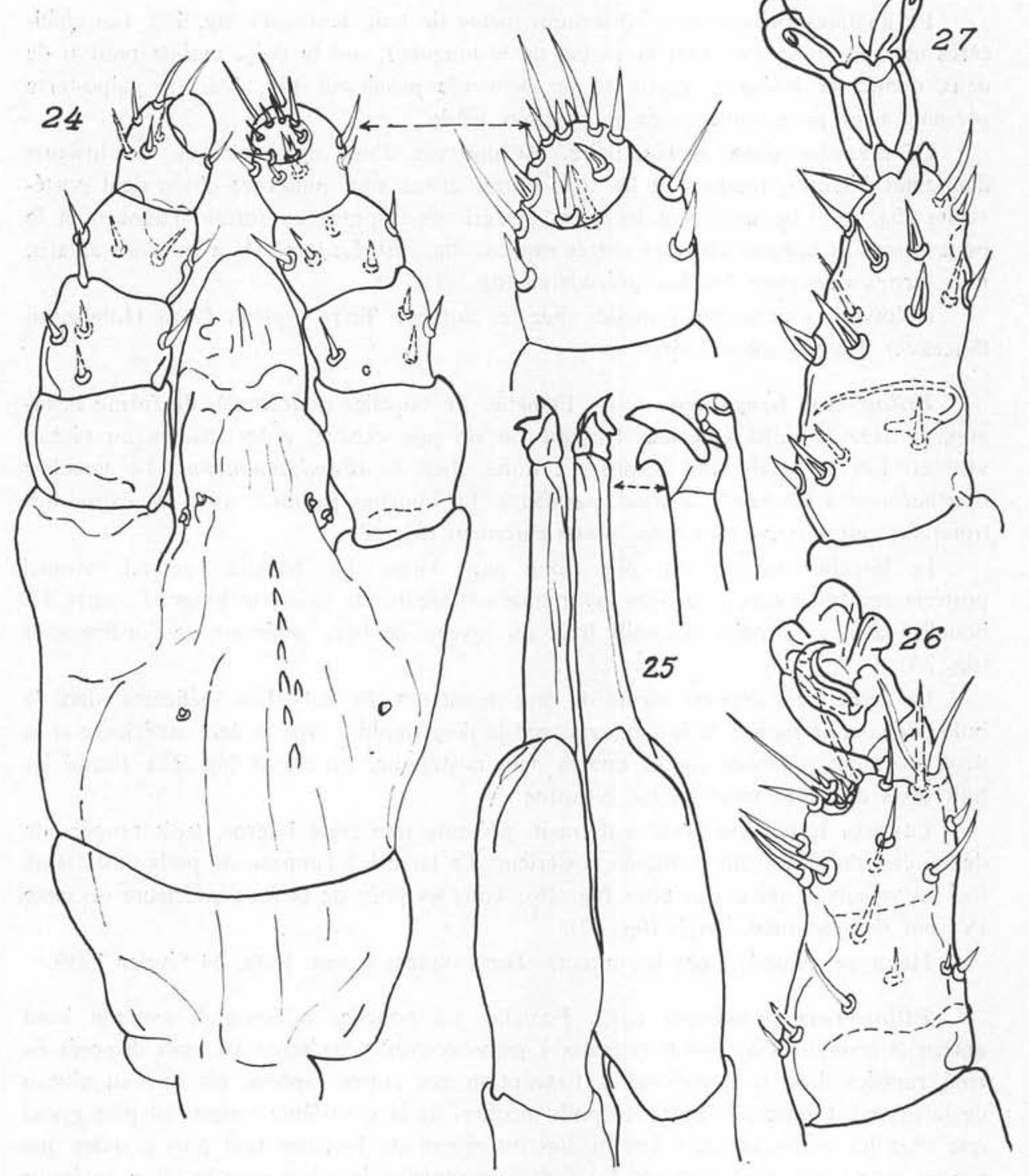

Planche X. - Ptilonyssus bregetovae n. sp., femelle

Fig. 24. - Gnathosoma, vue ventrale

FIG. 25. - Chélicères

FIG. 26. - Tarse I

Fig. 27. - Tarse IV 
poils fixée un peu en avant de l'anus, a le cribum pourvu de denticules moins nombreuses (fig. 16).

Le gnathosoma a le deutérosternum formé de huit denticules (fig. 17). Les chélicères avec le bulbe mesurant la moitié de la longueur, ont le doigt mobile pourvu de deux denticules latéraux, égaux et un denticule postérieur (fig. 18). Le palpo-tarse présente neuf poils spiniformes et un poils bifide.

La première coxe, rectangulaire, est pourvue d'une crête oblique et plusieurs denticules internes, tandis que les trois autres coxae sont pourvues d'une dent postérieure (fig. 19). Le tarse I a les poils postérieurs disposés en forme d'anneau et le bouton sensitif comme chez les autres espèces (fig. 20). Le tarse IV a les poils aplatis, plus étroits que chez l'espèce précédente (fig. 21).

Holotype et paratype, femelle, chez les auteurs. Terra typica: Gura Humorului (Suceava). Date 6 mars 1966.

Ptilonyssus bregetovae n. sp. Femelle. Le bouclier podosomal, de forme hexagonale, avec le bord antérieur tronqué ou un peu excavé, a les mailles du réseau visibles. Les 18 poils sont disposés comme chez $P$. strandtmannianus. Le bouclier opisthosomal a les bords latéraux parallèles. Le bouclier pygidial, avec les extrémités tronquées, est à peine plus large postérieurement (fig. 22).

Le bouclier sternal est plus long que large. Le bouclier génital, aminci postérieurement, est pourvu, vers l'extrémité antérieure, de striations longs et courts. Le bouclier anal a la paire de poils fixée au niveau de tiers antérieur de l'orifice anal (fig. 23).

Le deutérosternum est formé de sept denticules (fig. 24). Les chélicères, dont le bulbe dépasse la moitié de la longueur, ont le doigt mobile avec la dent antérieure et la dent moyenne courbées, tandis que la dent postérieure est droite (fig. 25). Parmi les huit poils du palpo-tarse un est bifurqué.

La coxa I, rétrécie postérieurement, présente une crête interne, trois rangées de denticules internes et un denticule postérieur. Le tarse I a l'anneau de poils postérieurs fixé au voisinage des autres poils (fig. 26). Tous les poils de la face inférieure du tarse IV sont moindrement élargis (fig. 27).

Halotype, femelle, chez les auteurs. Terra typica, Sinaia. Date, 24 février 1959.

Ptilonyssus pelmaspis n. sp. Femelle. Le bouclier podosomal, avec le bord antérieur arrondi et les bords latéraux à peine convexes, présente 16 poils disposés en cinq rangées dont le quatrième, à l'exception des autres espèces, est fixé au niveau de la moitié. L'intervalle entre les poils médians de la quatrième rangée est plus grand que chez les autres espèces. Les mailles du réseau du bouclier sont plus grandes que sur les autres boucliers dorsaux. La forme de semelle de sabot avec le talon antérieur est caractéristique pour le bouclier opisthosomal. Les bords du bouclier pygidal, de forme triangulaire, sont irréguliers (fig. 28).

Le bouclier sternal, piriforme, est élargi postérieurement. Le bouclier génital, avec les bords latéraux presque parallèles et le bord antérieur droit, est précédé d'un 


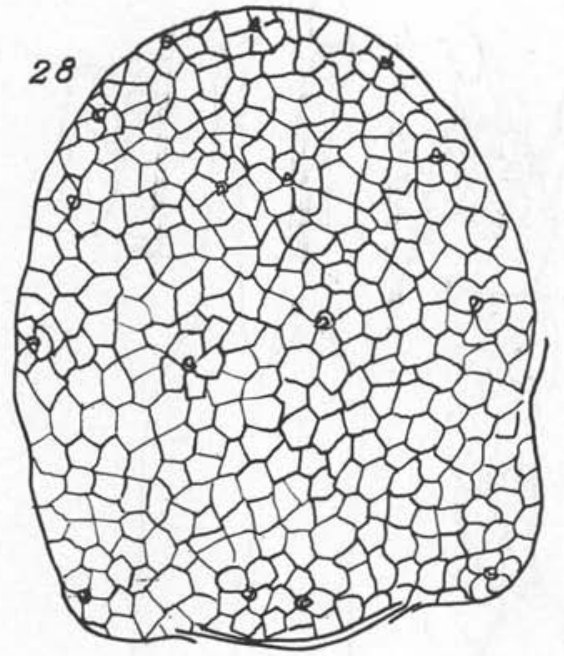

PLANCHE XI

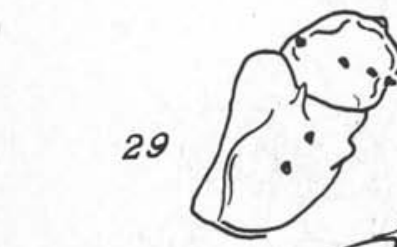

ح

\%8.
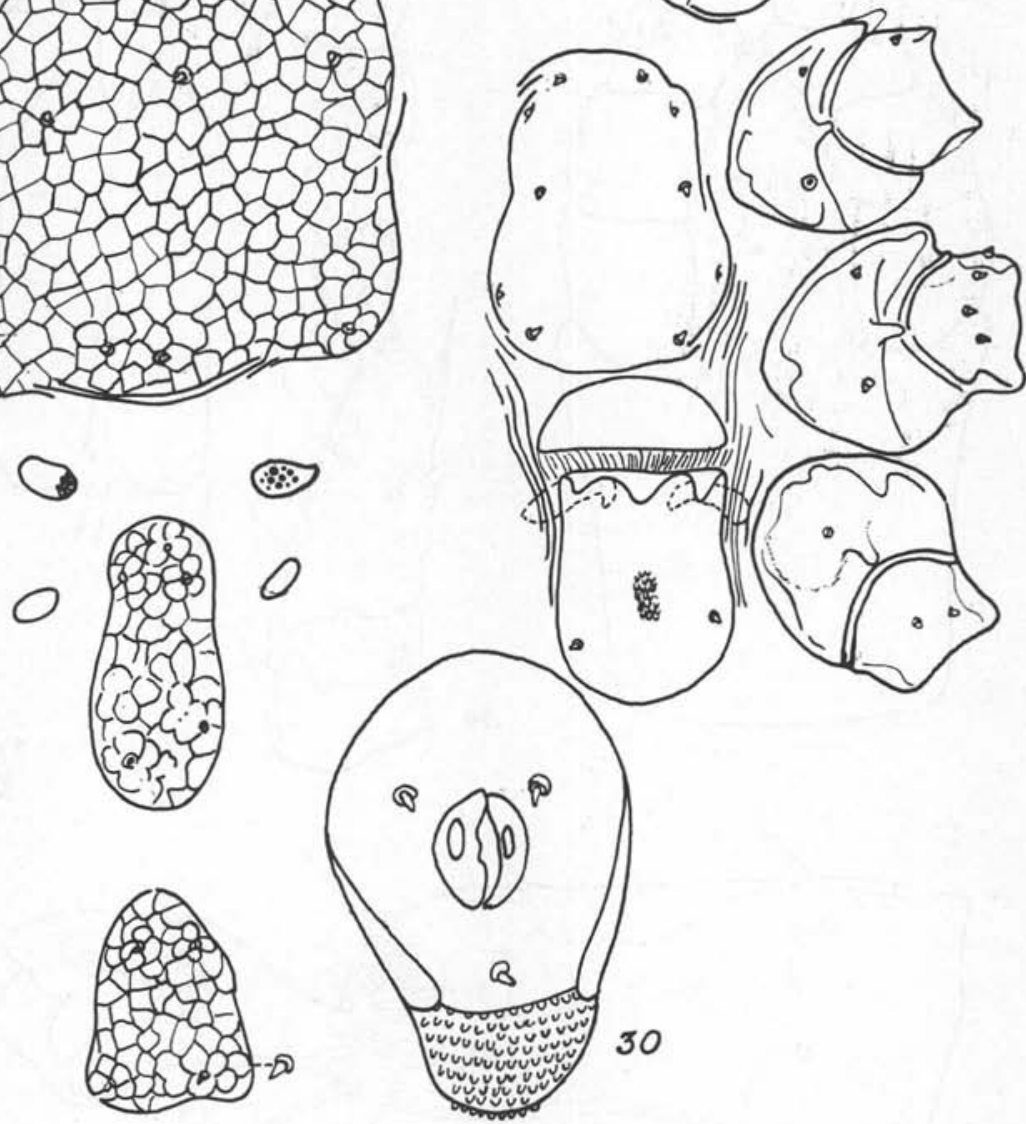

Planche XI. - Ptilonyssus pelmaspis n. sp., femelle

Fig. 28. - Boucliers dorsaux

Fig. 29. - Bouclier génital, sternal et coxes

Fig. 30. - Bouclier anal 


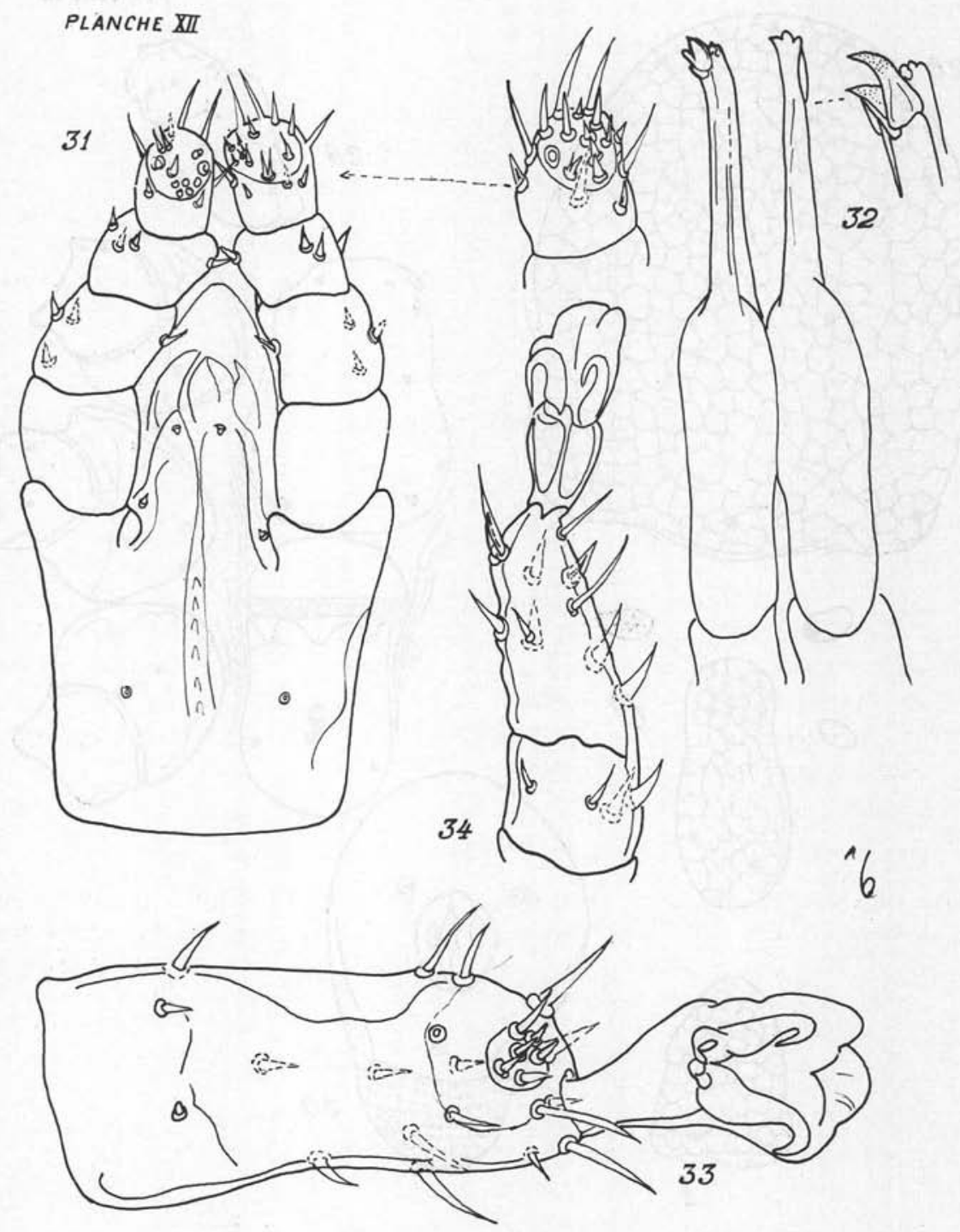

Planche XII. - Ptilonyssus pelmaspis n. sp., femelle

FIG. 31. - Gnathosoma, vue ventrale

FIG. 32. - Chélicères

FIG. 33. - Tarse I

Fị. 34. - Tarse IV 
bouclier de forme irrégulier qui le sépare du bouclier sternal (fig. 29). Le bouclier anal, triangulaire, avec les angles arrondis, a la paire de poils fixée un peu en avant de l'orifice anal (fig. 30).

Le deutérosternum est formé seulement de six denticules (fig. 31). Les chélicères, dont le bulbe est sensiblement plus long que la partie antérieure, ont le doigt mobile pourvu de deux denticules, dont l'antérieur est plus courbé (fig. 32). Parmi les 11 poils du palpo-tarse, cinq sont très petits et un est bifide (fig. 31 ).

Les coxae, dont la première est trapézoïdale, rétrécie médialement et les autres de forme demi-lunaire, sont dépourvues d'épines (fig. 29). L'anneau proximal des poils du tarse I n'est pas isolé des autres poils (fig. 33). Les poils de la face inférieure du tarse IV sont à peine aplatis (fig. 34).

Halotype, femelle, chez les auteurs. Terra typica, Sinaia. Date, 24 février 1959.

Clé dichotomique pour les espèces de Ptilonyssus parasites sur la sittelle. Femelle.

1.(2.) Bouclier opisthosomal fragmenté ................ Ptilonyssus tribaspis

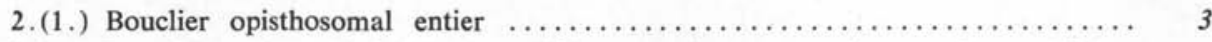

3. (4.) Bouclier podosomal, à bords parallèles, avec le rapport plus grand que $1,30 \ldots \ldots \ldots$

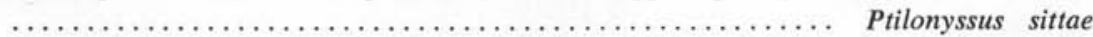

4. (3.) Bouclier podosomal, à bords convexes ou presque convexes, avec le rapport plus petit

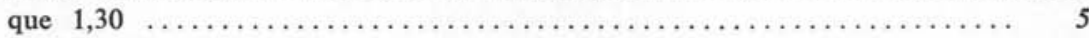

5.(6.) Bouclier opisthosomal en forme de semelle de sabot ...... Ptilonyssus pelmaspis

6.(5.) Bouclier opisthosomal ayant une autre forme $\ldots \ldots \ldots \ldots \ldots \ldots \ldots \ldots \ldots, 7$

7.(8.) Bouclier opisthosomal biffide ............... Ptilonyssus strandtmannianus

8.(7.) Bouclier opisthosomal entier .................. Ptilonyssus bregetovae

\section{Bibliographie}

1. Bregetova (G. N.), 1964. - Some problems of evolution of the Rhinonyssid mites, Report presented at the first International Congress of Parasitology. Nauka, Leningrad.

2. FAIN (A.), 1956. - Les Acariens de la famille Rhinonyssidae, Vitzthum, 1935, parasites des fosses nasales des Oiseaux au Ruanda-Urundi, Rev. Zool. Bot. Afr., LIII 1-2, p. 131-157.

3. —, 1964. - Les Acariens parasites nasicoles des Oiseaux de Belgique, IV, Bull. Ann. Soc. R. Ent. Belg., t. $100, \mathrm{n}^{\circ} 3$, p. 55-61.

4. - 1964. - Notes sur quelques Acariens parasites des voies respiratoires d'Oiseaux sud-américains (Rhinonyssidae: Mesostigmata), Bull. Soc. R. Zool. Anvers, $\mathrm{n}^{\circ} 33$, p. 3-12.

5. - 1966. - Les Acariens parasites nasicoles des Oiseaux de Belgique, V, Bull. Ann. Soc. R. Ent. Belg., t. 102, $\mathrm{n}^{\circ}$ 6, p. 117-122.

6. - et AitKen (T.H. G.), 1967. - Les Acariens parasites nasicoles des Oiseaux de Trinidad (Indes Occidentales), I, Rhinonyssidae: Mesostigmates, Bull. Inst. R. Sci. Nat. Belg., 43, 12, p. 1-44.

7. Strandtmann (R. W.), 1960. - Nasal mites of Thailand birds (Acarina: Rhinonyssidae), Jour. Kansas Ent. Soc., t. 33, $\mathrm{n}^{\circ} 4$, p. 129-151. 